\title{
Experimental and computational investigation of binary drop collisions under elevated ambient pressure
}

\author{
Louis Reitter ${ }^{1}$, Muyuan Liư ${ }^{2}$, Jan Breitenbach ${ }^{1 *}$, Kuan-Ling Huang ${ }^{3}$, Dieter Bothe ${ }^{2}$, \\ Günter Brenn ${ }^{4}$, Kuo-Long Pan ${ }^{3}$, llia V. Roisman ${ }^{1}$, Cameron Tropea ${ }^{1}$ \\ ${ }^{1}$ Fluid Mechanics and Aerodynamics, TU Darmstadt, Germany \\ ${ }^{2}$ Mathematical Modeling and Analysis, TU Darmstadt, Germany \\ ${ }^{3}$ Mechanical Engineering, National Taiwan University, Taiwan \\ ${ }^{4}$ Fluid Mechanics and Heat Transfer, TU Graz, Austria \\ ${ }^{*}$ Corresponding author: jbreitenbach@sla.tu-darmstadt.de
}

\begin{abstract}
Spray systems often operate under extreme ambient conditions like high pressure, which can have a significant influence on important spray phenomena. One of these phenomena is binary drop collisions. Such collisions, depending on the relative velocity and the impact parameter (eccentricity of the collision), can lead to drop bouncing, coalescence or breakup. This experimental and computational study is focused on the description of the phenomenon of drop bouncing, which is caused by a thin gas layer preventing the drops coalescence. To identify the main influencing parameters of this phenomenon, experiments on binary drop collisions are performed in a pressure chamber. This experimental system allows us to investigate the effect of an ambient pressure (namely the density and viscosity of the surrounding gas) on the bouncing/coalescence threshold.
\end{abstract}

\section{Keywords}

drop collision, breakup/coalescence, multiphase flow

\section{Introduction}

Understanding the collisions of drops is of interest for many fields of application. They play an important role in raindrop formation [1, 2] and in the modeling of fuel combustion [3, 4]. Systematic experimental studies visualizing binary drop collisions provide information allowing to construct regime maps of the collision outcomes [5, 6, 7]. The mechanisms of bouncing, coalescence, stretching separation, reflexive separation, and shattering are observed. These mechanisms are determined by the kinetic and geometrical parameters of the collision, as well as the properties of the drop liquids and the surrounding gas. The main kinetic and geometrical parameters are defined in Figure 1, where $D_{1}$ and $D_{2}$ are the drop diameters and $V$ is the relative drop velocity. $B$ is the distance of closest approach of the drop centers, measured orthogonal to $V$ at the instance of the collision. Dimensional analysis reveals that the collision Weber number We $=V^{2} D_{1} \rho / \sigma$, the Ohnesorge number $\mathrm{Oh}=\mu / \sqrt{\sigma D_{1} \rho}$, the impact parameter $X=2 B /\left(D_{1}+D_{2}\right)$ and the drop size ratio $\Delta D=D_{1} / D_{2}$ determine the outcome of the collision, if the medium ambient to the colliding drops is not varied.

The present investigation concentrates on the mechanism of bouncing. Outcomes from drop collsion with a solid substrates shown, that small droplets are able to bounce on solid substrate, irrespectivly of their wettability [8]. The bouncing is caussed by a thin squeezing air film beetween the droplet and the substrate leading to squeeze forces. For the binary drop collsion, Rayleigh [9] pointed out that the drop collision mechanism may be influenced by electrical charge on the drops. The occurrence of bouncing or coalescence of electrified drops may depend on their charge density. Electrically charged drops formed from vertically upward pointing electrified liquid jets may either coalesce (at moderate charge density) or rebound (at high charge density).

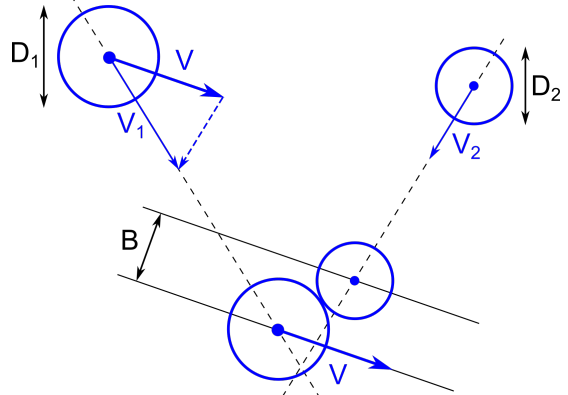

Figure 1. Definition of collision geometry This behaviour determines the appearance of the jet, which may be either fairly coherent or strongly scattered, respectively. Qian and Law [7] carried out experiments in atmospheres of different gases at different pressures, showing that the extension of the bouncing regime in the collision charts varies with the ambient gas pressure. At higher gas pressure, the bouncing regime widens. The experimental study [10] demonstrates that the bouncing regime relies on the presence of the ambient gas. The regime disappears if the drops collide in a vacuum. This important result clearly indicates the role of the medium ambient to the colliding drops in the occurrence of bouncing. Consequently, the study [11] shows that bouncing is enhanced with drops colliding in another viscous fluid.

From the literature we know several models for binary drop collision outcomes applied in the framework of CFD codes, such as the KIVA-II code. The first model for bouncing was presented in [12]. It assumes that, upon impact, 
the drops deform into semi-spherical shapes. If the deformation produces an increase in surface energy larger than the initial kinetic energy of the pair, the drops will bounce. The resulting criterion is a threshold of the impact Weber number as a function of the impact parameter and drop size ratio. The model is empirical, built on collision charts, and does not represent the flow details in the space between the approaching drops [13]. This model is used by the group of Reitz to predict drop collision outcomes, accounting for all the four mechanisms at moderate Weber numbers, except shattering [14]. The conclusion is that, in the present literature, we do not see any detailed investigation of the flow in and around colliding drops for the bouncing mechanism.

The effect of the ambient pressure on the thresholds between the regimes is not yet completely understood. The main subject of this study is the experimental and computational investigation of binary drop collisions under elevated ambient pressure with emphasis on bouncing. The goal is to better understand this mechanism caused by the viscous air flow in a very thin gap between the colliding drops, and to model the transition conditions between coalescence and bouncing. The regime maps allow the threshold Weber number between bouncing and coalescence to be determined as a function of the impact parameter. One important result of this study is that only a minor dependence of the drop coalescence on the elevated ambient pressure is observed. It should be noted that, for high pressures, the value of the kinematic viscosity of air is almost constant.

\section{Experimental and Computational Methods \\ Experimental setup}

The experimental setup consists of two drop generators mounted in a pressure chamber, a high-speed video system and a data acquisition system. The scheme of the setup is shown in Figure 2. The pressure chamber can be operated in the range of absolute pressure from 1 bar to 20 bar. It has four glass windows to provide optical access to the studied phenomena.

Two drop generators are used to generate a chain of drops and can be controlled independently. They are connected to a pressurized fluid supply tank and a signal generator. The electrical signal powers the drop generators' piezo actuators, which are used to impose disturbances onto the continuous fluid jets. If the disturbance frequency is properly set, the jet undergoes a controlled Rayleigh-type breakup and a stream of monodisperse drops is produced. The drop diameter can be varied by the disturbance frequency and by changing the orifice diameter of the drop generator.

Two drop generators are positioned in front of one chamber window. For precise positioning, one drop generator is mounted on a manual micromanipulator and the other drop generator is mounted on a highprecision traversing system. The piezo motor of the traversing system can adjust one drop generator position in $5 \mu \mathrm{m}$ steps. This fine adjustment of the drop generator position allows to ensure equal distances between each drop generator and the camera objective. The high-speed camera is operated with a frame rate of 22000 frames per second. Continuous illumination using an LED light source is used in the experiments. A data acquisition and control system is used to control the fluid flow rate through the drop generators and to measure the experimental properties. These are the air pressure, temperature and relative humidity in the pressure chamber, the supply fluid temperature and the pressure in the fluid reservoir. The relative velocity of the colliding drops, their diameters and impact parameter are calculated via image processing.

In the experiments $42 \mathrm{wt} . \%$ glycerol-water solution at a

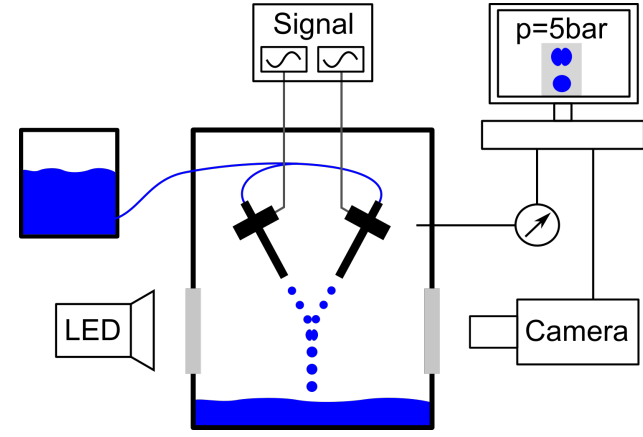

Figure 2. Scheme of the experimental setup

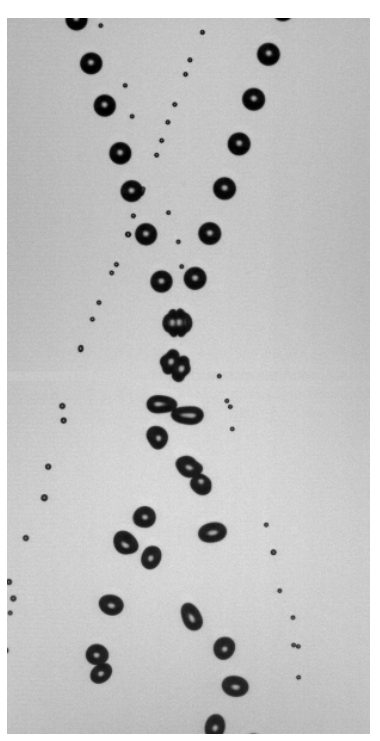

(a) bouncing

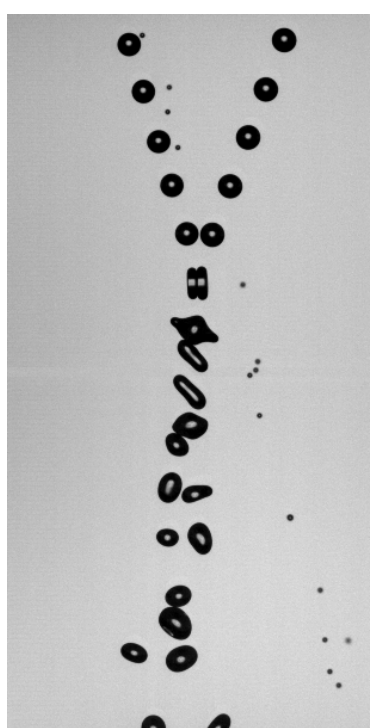

(b) coalescence
Figure 3. Exemplary observations of binary drop collisions leading to bouncing or coalescence. temperature of $T_{f}=28^{\circ} \mathrm{C}$ is used for the drops, whose diameter ranges from $D_{1}=335-400 \mu \mathrm{m}$ with a constant drop size ratio $\Delta D \approx 1$. The impact velocity varied from $V_{1 / 2}=1.5-3.3 \mathrm{~m} / \mathrm{s}$. The dynamic viscosity, surface tension coefficient and density of the liquid are $\mu_{L}=3.25 \times 10^{-3}$ Pa.S, $\sigma=68.44 \times 10^{-3} \mathrm{~N} / \mathrm{m}$ and $\rho_{L}=1058 \mathrm{~kg} / \mathrm{m}^{3}$, respectively. The corresponding Ohnesorge number $\mathrm{Oh} \approx 0.02$ is almost constant in all the experiments. The air temperature in the pressure chamber was $T_{0}=28^{\circ} \mathrm{C}$ and the relative humidity $\phi=78 \%$.

Exemplary observations of drop collisions leading to different outcomes, bouncing or coalescence, are shown in Figure 3 . These images are then processed to measure the drops sizes and the impact velocity and to identify the 


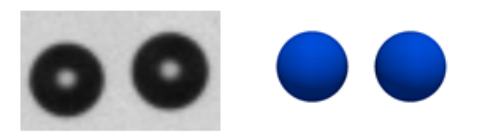

$t=0 \mathrm{~ms}\left(t^{*}=-0.379\right)$
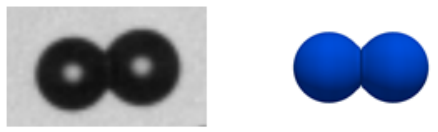

$0.091 \mathrm{~ms}(0.102)$
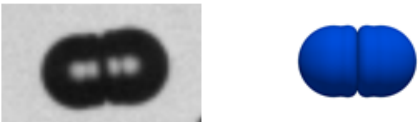

$0.136 \mathrm{~ms}(0.343)$
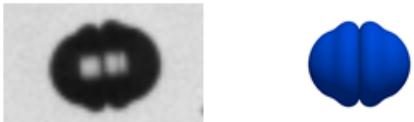

$0.182 \mathrm{~ms}(0.584)$
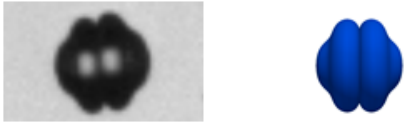

$0.227 \mathrm{~ms}(0.824)$
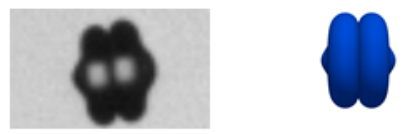

$0.273 \mathrm{~ms}(1.065)$

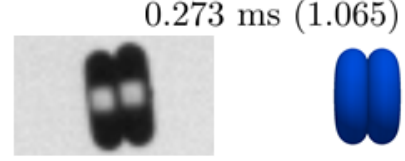

$0.318 \mathrm{~ms}(1.305)$
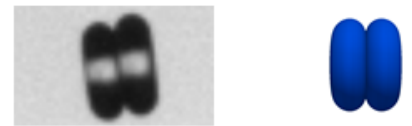

$0.364 \mathrm{~ms}$ (1.546)
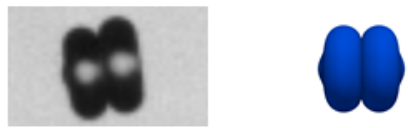

$0.409 \mathrm{~ms}(1.787)$
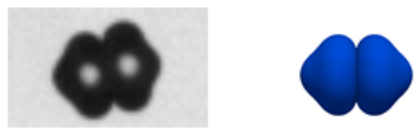

$0.455 \mathrm{~ms}(2.027)$
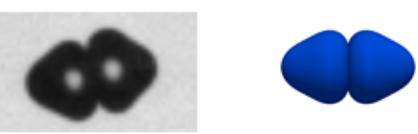

$0.500 \mathrm{~ms}(2.268)$
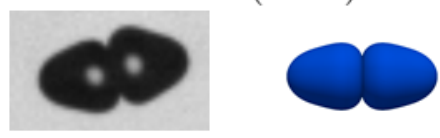

$0.546 \mathrm{~ms}(2.508)$
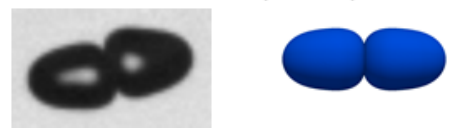

$0.591 \mathrm{~ms}(2.749)$
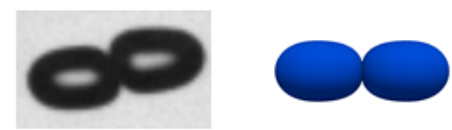

$0.636 \mathrm{~ms}(2.990)$
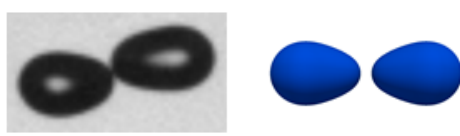

$0.773 \mathrm{~ms}(3.471)$

Figure 4. Drop bouncing near the coalescence threshold. Comparison of the computed drop shapes after axisymmetric drop collision with the experimental observations. The parameters are: $\mathrm{Re}=204.92$, We $=17.76, X=0.0135$, the pressure is $p=7$ bar. The three columns compare experiments (left) with numerical simulations (right).

main collision regimes. The two drop chains in the background are produced in the jet breakup region. Due to the high ambient pressure small satellite drops bounce off the main drop chain. These pre-collision droplets do not affect the binary collision.

\section{Computational method}

In order to better understand the mechanisms of binary drop collisions, this phenomenon is simulated numerically, solving the full Navier-Stokes equations in the liquid and gas phases and accounting for surface tension effects. The numerical simulations are performed with the in-house code Free Surface 3D (FS3D), which was originally developed at the ITLR (University of Stuttgart) [15] and has been extended both at the ITLR and in the Mathematical Modeling and Analysis (MMA) group of the Technische Universität of Darmstadt. FS3D is based on the Volume-ofFluid (VOF) method and solves the incompressible transient two-phase flow in 3D on a staggered Cartesian mesh. A recent overview of FS3D is given in [16]. FS3D has been validated in $[15,17,18,19]$ for the simulation of binary droplet collisions.

In FS3D, for integrating the surface tension force, the balanced-CSF [20] and CSS models [21] are implemented. The balanced-CSF model has the advantage of much less parasitic currents. However, it is not able to deal with the topology change when merging of two interfaces occurs. The CSS model can deal with the topology change in a natural manner; however, it suffers from parasitic currents. Based on their advantages and disadvantages, the balance-CSF model is used before possible coalescence in the simulations, while at the instant of coalescence, the computation of the surface tension force is computed according to the CSS model.

In experiments, prior to possible coalescence, there is a gas layer between the colliding droplets with a thickness generally of the order of a few $10 \mathrm{~nm}$ [22]. The gas layer cannot be resolved due to current restrictions in computational effort. This results in a numerical coalescence of droplets in standard VOF simulations, since the computation of the surface tension force of one droplet is affected by its counterpart. In order to simulate bouncing, specifically in case of a symmetric collision, we temporarily remove the symmetric counterpart of one droplet while computing the surface tension force. This treatment is also valid for the case of coalescence before the merging occurs. At the instant of possible coalescence, this treatment is discarded so that coalescence then occurs. The time instant of coalescence is defined as the averaged value of the instants that correspond to the last cuspy contour and the first smooth contour between the two droplets [23], which are obtained from the experiment.

In the simulations, one droplet with diameter $D_{1}$ is initialized, colliding towards its symmetric counterpart with velocity $V$. A rectangular box with the dimensions of $\left(2 D_{1}\right)^{3}$ is discretized with $256^{3}$ equidistant Cartesian cells. Three symmetry planes are employed for reducing the computational effort. Slip conditions are prescribed on the symmetry planes while homogeneous Neumann boundary conditions for the velocity and zero pressure are imposed on the other parts of the boundary. 


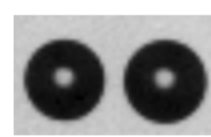

$t=0 \mathrm{~ms}\left(t^{*}=-0.0002\right)$

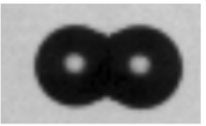

$0.091 \mathrm{~ms}(0.434)$

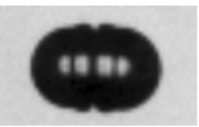

$0.136 \mathrm{~ms}(0.651)$
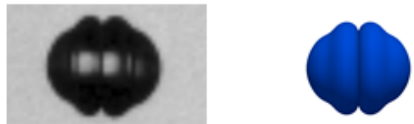

$0.182 \mathrm{~ms}(0.869)$

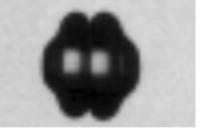

$0.227 \mathrm{~ms}(1.086)$

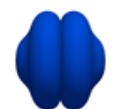

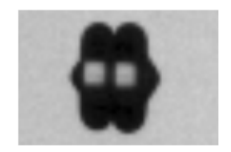

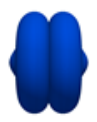

$0.273 \mathrm{~ms}(1.303)$
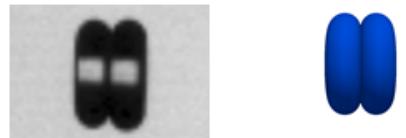

$0.318 \mathrm{~ms}(1.520)$
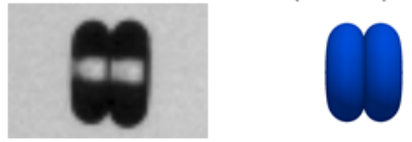

$0.364 \mathrm{~ms}(1.737)$
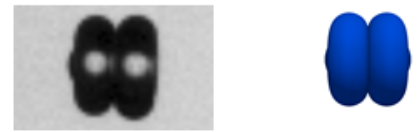

$0.409 \mathrm{~ms}(1.955)$
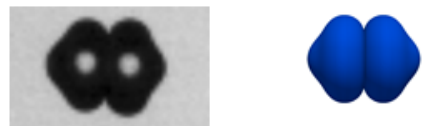

$0.455 \mathrm{~ms}(2.172)$
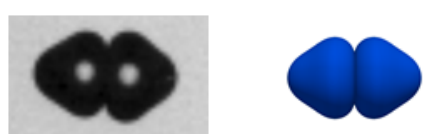

$0.500 \mathrm{~ms}(2.389)$
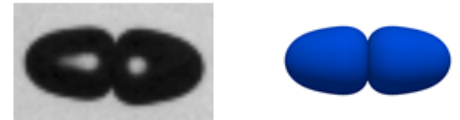

$0.590 \mathrm{~ms}(2.824)$
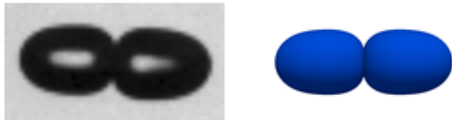

$0.636 \mathrm{~ms}(3.041)$
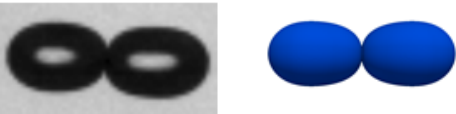

$0.682 \mathrm{~ms}(3.258)$
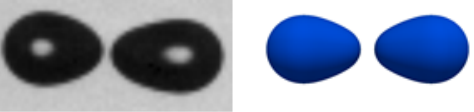

$0.773 \mathrm{~ms}(3.692)$

Figure 5. Drop bouncing. Comparison of the computed drop shapes after axisymmetric drops collision with the experimental observations. The parameters are: $\operatorname{Re}=208.10, \mathrm{We}=17.27, X=0.005$, the pressure is $p=9$ bar.
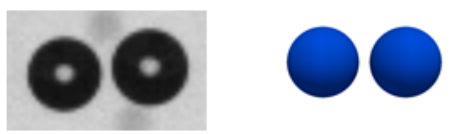

$t=0 \mathrm{~ms}\left(t^{*}=-0.0002\right)$
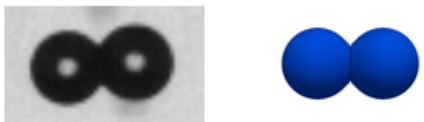

$0.045 \mathrm{~ms}(0.242)$
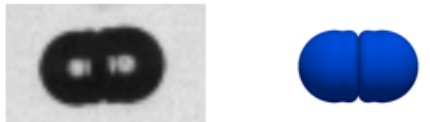

$0.091 \mathrm{~ms}(0.484)$
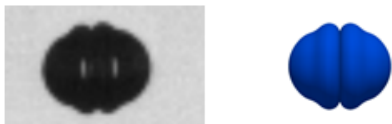

$0.136 \mathrm{~ms}(0.726)$
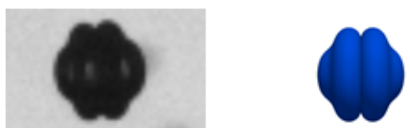

$0.182 \mathrm{~ms}(0.968)$
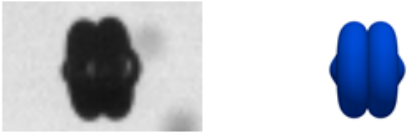

$0.227 \mathrm{~ms}(1.209)$
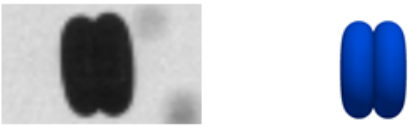

$0.272 \mathrm{~ms}(1.451)$
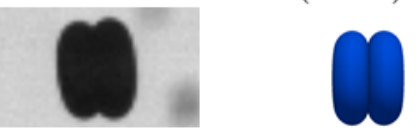

$0.318 \mathrm{~ms}(1.693)$
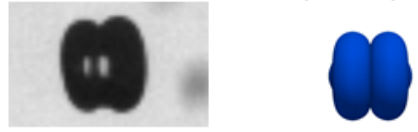

$0.364 \mathrm{~ms}(1.935)$

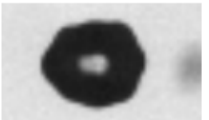

$0.409 \mathrm{~ms}(2.177)$
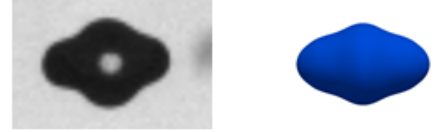

$0.455 \mathrm{~ms}$ (2.419)
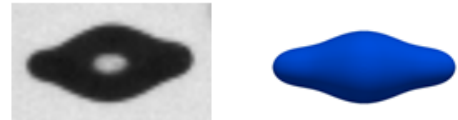

$0.591 \mathrm{~ms}(3.145)$
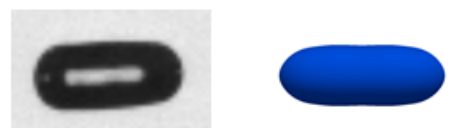

$0.727 \mathrm{~ms}(3.871)$
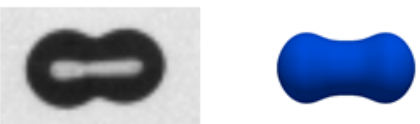

$0.864 \mathrm{~ms}(4.596)$

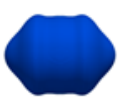

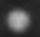

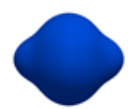

$1.136 \mathrm{~ms}$ (6.048)

Figure 6. Drop coalescence. Comparison of the computed drop shapes after axisymmetric drops collision with the experimental observations. The parameters are: $\operatorname{Re}=210.01, \mathrm{We}=18.48, X=0$, the pressure is $p=7$ bar. The coalescence is imposed in the computations. 


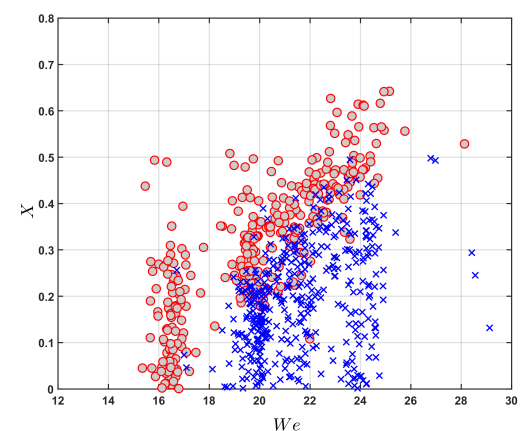

(a)

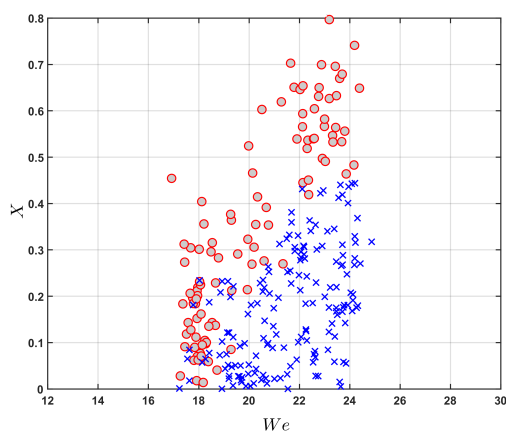

(b)

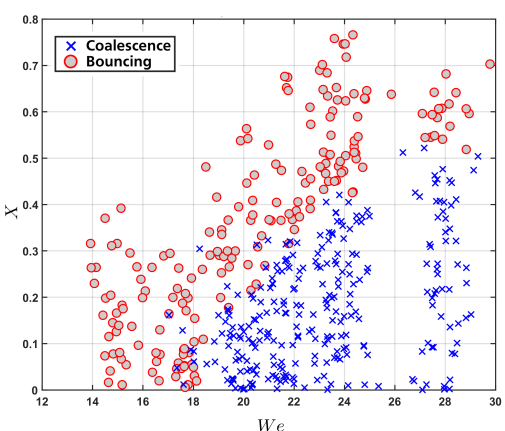

(c)

Figure 7. Bouncing-coalescence diagrams of the experimental outcome for various Webern numbers We and impact parameters $X$ at different ambient pressures: (a) $p=5.3$ bar, (b) $p=7.0$ bar, and (c) $p=9.0$ bar.

\section{Results and discussion}

The theoretically predicted shapes of the drops during collision are compared with the experimental data in Figures 4 - 6. The agreement is rather good for both bouncing cases (Figs. 4 and 5) and the coalescence case (shown in Figure 6). It should be noted, that since the coalescence still cannot be described numerically, the instant of coalescence during drop deformation was externally imposed. Some deviation between the predictions and experimental data at the later stages of drop coalescence can be explained by the sensitivity of the phenomena to the instant of coalescence (which is not known a priori).

The outcome from the binary drops collision is determined by many factors, like gas density, the impact Weber number and the impact parameter, which determine the duration of contact. The duration $t_{c}$ of the binary collision in the bouncing regime is governed by inertia and surface tension. It has been shown [10] that for high Weber numbers this time is scaled very well by half of the Rayleigh period of oscillation,

$$
t_{c}=A \sqrt{\frac{\pi^{2} \rho_{L} D_{1}^{3}}{8 \sigma}}
$$

where $A \approx \mathrm{We} \mathrm{e}^{0.043}$ is obtained by fitting to the experimental data reported in [10], $\rho_{L}$ and $\sigma$ are the liquid density and surface tension. It should be noted that in the case of drop rebound the contact time will be different, since the rim experiences an additional force associated with two liquid interfaces bounding the air gap. Therefore, the estimated corrected contact time is

$$
t_{c} \approx \mathrm{We}^{0.043} \sqrt{\frac{\pi^{2} \rho_{L} D_{1}^{3}}{16 \sigma}}
$$

for rebound. For the set of parameters corresponding to the experiments shown in Figures 4 and 5 , the contact time predicted by (2) is $t_{c}=0.708 \mathrm{~ms}$ and $t_{c}=0.772 \mathrm{~ms}$, respectively, which agrees rather well with the observed contact times. In any case, since the Weber number enters equation (2) only with a small power, we can assume

$$
t_{c} \sim \sqrt{\frac{\rho_{L} D_{1}^{3}}{\sigma}}=\frac{D_{1}}{V_{n}} \mathrm{We}^{1 / 2}
$$

for a certain range of Weber numbers.

The bouncing-coalescence diagrams for different ambient pressures, showing the types of collision outcome for various impact parameters $X$ and Weber numbers We, are shown in Figure 7. It is interesting that these diagrams for elevated pressures are very similar. This counter-intuitive result is explained by the fact that the influence of the pressure on the dynamic viscosity $\mu_{A}$ of the air in the gap is only minor. It indicates also that the viscosity effects in the gap between the drops are dominant. It is therefore obvious that the Weber number is not a relevant dimensionless group for description of the bouncing/coalescence threshold.

To develop a reliable expression for this threshold an accurate analysis of the air flow in the gap between the drop and further its stability analysis are necessary. In this study only rough estimations of the relevant parameters are performed. The typical air gap thickness $h_{\text {air }}$ between the drops can be roughly estimated using the lubrication approximation in the description of the gas flow and using the expression for the pressure $p$, produced by drop impact [24], i.e.

$$
p \sim \rho_{L} V_{n}^{2} \exp \left[-b \frac{t V_{n}}{D_{1}}\right], \quad h_{a i r} \sim\left[\frac{\mu_{A} D_{1}}{\rho_{L} V_{n}}\right]^{1 / 2}=D_{1} \operatorname{Re}_{A}^{-1 / 2}
$$

where $V_{n} \equiv V \sqrt{1-X^{2}}$ is the normal component of the relative impact velocity, $\mu_{A}$ is the air dynamic viscosity, $b$ is a dimensionless constant ( $b=3.1$ found from the computations in [24]), $\operatorname{Re}_{A}$ is the Reynolds numbers based on the air density and normal velocity component $V_{n}$. 


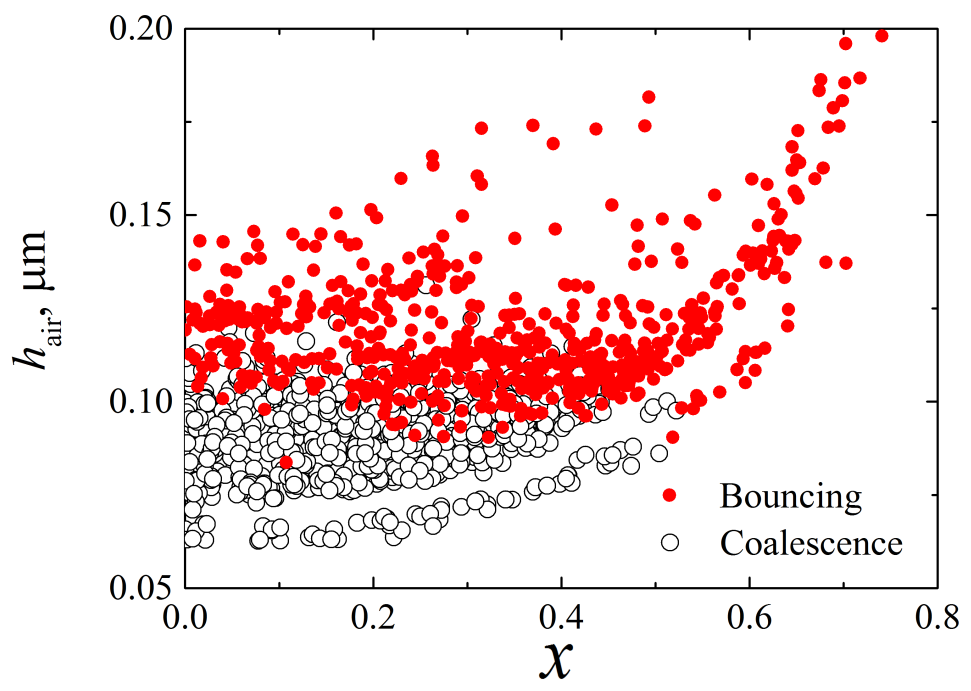

Figure 8. The predicted thickness of the air layer for bouncing and coalescence, roughly estimated in (5), as a function of the impact parameter.

For a long time after impact the thickness of the gap is governed by the flow in the lamellae. Such flow has been analyzed in [24, 25], leading to the evolution of the film thickness in the form $h \sim t^{-2}$. This analysis has been confirmed in the experiments with drop impact onto convex surfaces [26]. Finally, we estimate the thickness of the air layer during drop collision according to

$$
h_{\text {air }} \sim\left[\frac{\mu_{A} D_{1}}{\rho_{L} V_{n}}\right]^{1 / 2} \frac{D_{1}^{2}}{t_{c}^{2} V_{n}^{2}}=\frac{\mu_{A}^{1 / 2} \sigma}{\rho_{L}^{3 / 2} D_{1}^{1 / 2} / V_{n}^{5 / 2}}=D_{1} \operatorname{Re}_{A}^{-1 / 2} \mathrm{We}_{n}^{-1},
$$

where $\mathrm{We}_{n}$ is the Weber number based on the normal component of the impact velocity.

The dependence of the predicted values of $h_{\text {air }}$ on the dimensionless impact parameter $X$ for the cases of drop bouncing and coalescence is shown in Fig. 8. For small values of the impact parameter, $X<0.5$, the estimated minimum air gap thickness $h_{\text {air }}$ is approximately $100 \mathrm{~nm}$. Locally, the minimum gap thickness can be much smaller, since the gap thickness is strongly non-uniform and its value reduces near the rim area. Therefore, the physics of coalescence can depend on the intermolecular forces. Nevertheless, the value of $h_{\text {air }}$ is probably a relevant scale determining the coalescence threshold. Further analysis is still required to describe the mechanism of coalescence.

\section{Conclusions}

In this study, the bouncing and coalescence of two Newtonian drops in air under elevated pressure conditions have been observed using a high-speed camera. Additionally, the kinematics of drop deformation has been described numerically using a computational code based on the volume-of-fluid method. The typical times of contact are estimated and compared to existing expressions.

The thickness of the air gap between the drops is roughly estimated. It is shown that the predicted smallest gap thickness of the air gap is in our case approximately $100 \mathrm{~nm}$. This value can be used as a scale for the bouncing/coalescence threshold. However, more data are required for validation of this assumption and for development of a more precise model.

\section{Acknowledgements}

This research was supported by the the German Scientific Foundation (Deutsche Forschungsgemeinschaft) in the framework of the SFB TRR 75 Collaborative Research Center, subprojects C04 and A07. The author Louis Reitter has contributed to the present manuscript in the framework of the course "Sprays and Atomization".

\section{References}

[1] Brazier-Smith, P., Jennings, S. and Latham, J., Proc. R. Soc. Lond. A. 326(1566):393-408 (1972).

[2] Park, R., Ph.d. thesis, University of Wisconsin (1970).

[3] Faeth, G., Prog. Energy Combust. Sci. 3(4):191-224 (1977).

[4] O'Rourke, P. J. and Bracco, F. V., Proc. Inst. Mech. Eng. 9:101-106 (1980).

[5] Ashgriz, N. and Poo, J., J. Fluid Mech. 221:183-204 (1990).

[6] Jiang, Y., Umemura, A. and Law, C., J. Fluid Mech. 234:171-190 (1992).

[7] Qian, J. and Law, C., J. Fluid Mech. 331:59-80 (1997).

[8] de Ruiter, J., Lagraauw, R., Mugele, F. and van den Ende, D., J. Fluid Mech. 776:531-567 (2015). 
[9] Rayleigh, J., Proc. R. Soc. London 28:405-409 (1878).

[10] Willis, K. and Orme, M., Exp. Fluids 34(1):28-41 (2003).

[11] Kim, J. and Longmire, E. K., Exp. Fluids 47(2):263-278 (2009).

[12] Estrade, J., Carentz, H., Lavergne, G. and Biscos, Y., Int. J. Heat Fluid Flow 20:486-491 (1999).

[13] Post, S. and Abraham, J., Int. J. Multiphase Flow 28:997-1019 (2002).

[14] Munnannur, A. and Reitz, R., Int. J. Multiphase Flow 33:873-896 (2007).

[15] Rieber, M., Numerische Modellierung der Dynamik freier Grenzflächen in Zweiphasenströmungen, FortschrittBerichte VDI: Reihe 7, Strömungstechnik, VDI-Verlag, Düsseldorf (2004), PhD Thesis, Institut für Thermodynamik der Luft- und Raumfahrt (ITLR), Universität Stuttgart.

[16] Eisenschmidt, K., Ertl, M., Gomaa, H., Kieffer-Roth, C., Meister, C., Rauschenberger, P., Reitzle, M., Schlottke, K. and Weigand, B., Appl. Math. Comput. 272:508-517 (2016).

[17] Focke, C., Kuschel, M., Sommerfeld, M. and Bothe, D., Int. J. Multiph. Flow 56:81-92 (2013).

[18] Liu, M. and Bothe, D., J. Fluid Mech. 789:785-805 (2016).

[19] Planchette, C., Hinterbichler, H., Liu, M., Bothe, D. and Brenn, G., J. Fluid Mech. 814:277-300 (2017).

[20] Popinet, S., J. Comput. Phys. 228(16):5838-5866 (2009).

[21] Lafaurie, B., Nardone, C., Scardovelli, R., Zaleski, S. and Zanetti, G., J. Comput. Phys. 113(1):134-147 (1994).

[22] Zhang, P. and Law, C. K., Phys. Fluids 23(4):042102 (2011).

[23] Pan, K.-L., Law, C. K. and Zhou, B., Journal of Applied Physics 103(6):064901 (2008).

[24] Roisman, I. V., Berberović, E. and Tropea, C., Phys. Fluids 21(5):052103 (2009).

[25] Yarin, A. and Weiss, D., J. Fluid Mech. 283:141-173 (1995).

[26] Bakshi, S., Roisman, I. V. and Tropea, C., Phys. Fluids 19(3):032102 (2007). 